\title{
Right Lung, Middle Lobe, Medial Segment
}

National Cancer Institute

\section{Source}

National Cancer Institute. Right Lung, Middle Lobe, Medial Segment. NCI Thesaurus.

Code C132408.

The medial segment of the middle lobe of the right lung. 